\title{
Experimental study of Pomeron
}

\author{
A.Rostovtsev \\ Institute for Theoretical and Experimental Physics \\ Moscow, Russia
}

\begin{abstract}
A Pomeron phenomenon remains a mystery. A short review of the experimental situation in diffractive physics and an account of some spectacular manifestations of the Pomeron are given.
\end{abstract}

Keywords: Pomeron, Diffraction. 


\section{Introduction}

The notion of Pomeron円 has been introduced (Frautschi 1962) to term an unusual Regge pole suggested first by V.N.Gribov (1961) to describe main properties of particle scattering at asymptotically high energies. With advance of QCD in 70th the Pomeron phenomenology was vastly abandoned and the emphasis has been more on perturbative QCD effects. During the last decade the phenomenon of the Pomeron became again a hot subject both for theoretical and experimental scrutiny. This increased interest is mainly generated by data from Tevatron and HERA linking the Pomeron induced processes and the perturbative effects. These data and the success of QCD are important building blocks for construction of a microscopic theory of the Pomeron. It is believed, that such theory should also describe the confinement and QCD vacuum structure. This challenging task is far from being completed now and the present theoretical understanding the Pomeron is wired and contradictory. Below, I restrict myself to an account of the present experimental situation and some, to my view, spectacular experimental manifestations of the Pomeron. It is a particular honour for me to give this talk at ITEP, in the very building where I.Ya Pomeranchuk (the Russian physicist after whom the Pomeron was termed) has being working for a long time.

What do we (experimentalists) mean today by the "Pomeron"? The Pomeron is thought to be a colourless and flavourless extended object similar to a hadronic resonance. The Pomeronhadron similarity can be illustrated by comparison of properties of an elastic scattering reaction, say

$$
p+p \rightarrow p+p
$$

and a reaction with charge exchange

$$
\pi^{-}+p \rightarrow \pi^{0}+n
$$

In both reactions the colliding particles exchange something which carries a 4-momentum and specific quantum numbers. Regge theory says, that a cross section of these reactions is a simple function

$$
\frac{d \sigma}{d t} \sim s^{2 \alpha(t)-2}
$$

where $s$ and $t$ are total energy and momentum transfer squared. Figure 1 shows the measurements (Bolotov 1974, Akerlof 1976) of the $\alpha$ as function of $t$ for reactions (1) and (2). These measurements can be parameterized by a linear form, called trajectory

$$
\alpha(t)=\alpha(0)+\alpha^{\prime} t
$$

In case of the reaction (2) the $\rho$ is a resonance with correct quantum numbers exchanged in the reaction. If the spin of $\rho$ is plotted against the square of its mass on Fig. 1a one finds that extrapolation of the trajectory to positive values $t \equiv M^{2}$ goes through the $\rho$ point. Moreover, there is a rich family of resonances, which belong to this trajectory. This is a beautiful finding related to the analyticity of scattering amplitudes.

\footnotetext{
${ }^{1}$ Historically, it was first called "Pomeranchuk" Regge pole.
} 
The trajectory for elastic reaction has parameters which differ from those of $\rho$-trajectory. Namely, an intercept $\alpha_{\mathbb{P}}(0) \approx 1.1$ and a slope $\alpha_{\mathbb{P}}^{\prime} \approx 0.25 \mathrm{GeV}^{-2}$. This trajectory is called Pomeron trajectory and the object exchanged in the elastic reaction (11) is called Pomeron. It carries vacuum quantum numbers $(\mathrm{C}=\mathrm{P}=+1)$, and is thought to be like a gluonic bubble. Though, the Pomeron trajectory has no undebatable particles on it, it is widely agreed that, if they will be found, they are glueballs. The Pomeron trajectory plays an important role at high energies describing an universal rise of the total $p p, \pi p, K p, \gamma p$ cross sections (Donnachie 1992) and a shrinkage of the forward peak in diffractive processes.

Since the Pomeron is a colourless object it's signature in a high energy event is a void of particles spanning a large rapidity interval, so-called rapidity gap. Unlike fluctuations in gluon fragmentation the Pomeron induced rapidity gaps are not exponentially suppressed. A class of reactions with non-exponentially suppressed large rapidity gaps is operationally termed diffractive reactions (Bjorken 1994). It is convenient to classify the diffractive reactions by a position of large rapidity gap in the event. In diffractive reactions with a beam hadron staying intact the rapidity gap is adjacent to the direction of this beam particle. The reaction with rapidity gap adjacent to only one of the beams is termed single diffractive dissociation (SDD). The inelastic reaction with two rapidity gaps adjacent to the both beams is called Double Pomeron Exchange (DPE). The reaction with both beam particles dissociating in to hadronic systems and leaving a rapidity gap inbetween is called double diffractive dissociation (DDD).

An example of a measured by H1 (1995) rapidity gap width distribution is shown in Figure 2 in terms of $\eta_{\max }$ variable. With decreasing of the $\eta_{\max }$ the rapidity gap grows. One observes the exponentially falling spectrum flattens out for $\eta_{\max }<2$. In this region of large rapidity gaps the diffractive process dominates and can be experimentally separated from non-diffractive events. Why the rapidity gap spectrum for diffractive reaction is flat? The answer to this question points to a radiative type of the Pomeron emission by beam particles similar to radiation of photon. For experimentalists the picture suggested by Ingelman and Schlein (1985), is very illustrative. First, a Pomeron is emitted from one of beam particles with momentum transfer $t$ and probability $P \sim 1 / x_{\mathbb{P}}$, where $x_{\mathbb{P}}$ is a fraction of the beam energy taken by the Pomeron. Then, this Pomeron interacts inelastically with the other incoming beam particle. Assume, the Pomeron has typical hadronic properties, so that the system $X$, produced in a Pomeron-particle collision, would resemble normal minimum bias events in hadron-hadron inelastic scattering at corresponding invariant mass $M_{X}^{2}=x_{\mathbb{P}}\left(2 E_{\text {beam }}\right)^{2}$. This system decays in to final state hadrons with momentum distribution given by a longitudinal phase space. A rapidity interval filled by the final state hadrons is $\Delta \eta \sim \log \left(M_{X}^{2}\right)$, then

$$
\frac{d \sigma_{\text {diff }}}{d \Delta \eta} \sim M_{X}^{2} \frac{d \sigma_{\text {diff }}}{d M_{X}^{2}} \sim x_{\mathbb{P}} \frac{1}{x_{\mathbb{P}}} \sim \text { const }
$$

In a fraction of events resulting from the Pomeron-particle interaction high $P_{t}$ hadronic jets are produced. In the Ingelman-Schlein model the produced jets carry a direct information on the partonic content of the Pomeron. Following this approach, the DPE reaction is seen like a collision of two Pomerons. Interesting to note, though the resulting hadronic system is produced fully isolated in a rapidity space, and nevertheless, it is aligned with the initial beam direction.

The above described picture of diffraction assumes a factorization of Pomeron flux and the Pomeron-particle cross section. In order to make this picture quantitative it is practical to use the 
Pomeron flux predicted by the Regge theory. In a framework of Regge theory the cross section for a single diffractive dissociation resulting from one Pomeron exchange can be written (see e.g. Goulianos 1983) as

$$
\frac{d^{2} \sigma_{S D D}}{d x_{\mathbb{P}} d t}=\left[\frac{\beta^{2}(t)}{16 \pi x_{\mathbb{P}}^{2 \alpha_{\mathbb{P}}(t)-1}}\right] \times\left\{g \beta(0)\left(\frac{x_{\mathbb{P}} s}{s_{0}}\right)^{\alpha_{\mathbb{P}}(0)-1}\right\},
$$

where $\beta(t)$ is the coupling of the Pomeron to a beam hadron, $g$ is the triple-Pomeron coupling, $s_{0}$ is an arbitrary scale parameter. The function $\beta(t)$ is obtained from the elastic scattering experiment. The term in the square brackets is interpreted as a Pomeron flux, while the remaining factor is identified as a Pomeron-hadron total cross section. Further on the Pomeron flux defined by (3) is termed "standard Pomeron flux". The factorization formula (3) has correct $M_{X}^{2}$ behaviour

$$
\left.\frac{d^{2} \sigma_{S D D}}{d M_{X}^{2} d t}\right|_{t=0} \sim\left(\frac{1}{M_{X}^{2}}\right)^{1.1},
$$

measured in the experiments (Goulianos 1999). At the same time, the formula (3) implies fast rise of the diffractive cross section with energy

$$
\left.\frac{d^{2} \sigma_{S D D}}{d M_{X}^{2} d t}\right|_{t=0} \sim s^{2 \alpha_{\mathbb{P}}(0)-2},
$$

which is not supported by the experimental data discussed in the following section.

\section{Pomeron at HERA and Tevatron}

The measurements at HERA of deep-inelastic electron-proton scattering (DIS) have demonstrated that about $10 \%$ of events have large rapidity gap adjacent to the proton beam direction. A natural interpretation of these rapidity gap events is based on the hypothesis that highly virtual photon probes a Pomeron emitted from the proton. Therefore, at HERA one can measure a partonic structure of the Pomeron the same way as the proton structure function $F_{2}^{p}$ is measured. By analogy with the $F_{2}^{p}$ one defines the diffractive structure function $F_{2}^{D(3)}$

$$
\frac{d \sigma_{e p \rightarrow e p X}}{d x d Q^{2} d x_{\mathbb{P}}}=\frac{4 \pi \alpha^{2}}{x Q^{4}}\left(1-y+\frac{y^{2}}{2}\right) F_{2}^{D(3)},
$$

where $x, Q^{2}$ are usual variables used to describe DIS. The variable $x_{\mathbb{P}}$ is calculated in the experiment from the measured mass of hadronic system $M_{X}$ resulting from photon-Pomeron interaction

$$
x_{\mathbb{P}}=\frac{x}{\beta}, \beta \approx \frac{Q^{2}}{Q^{2}+M_{X}^{2}},
$$

where $\beta$ is a ratio of the momentum carried by the quark coupling to a virtual photon to that of the Pomeron. In another words, $\beta$ is an analog of $x$-Bjorken for the Pomeron. 
Figure 3 shows the measured by the H1 Collaboration (1997) $F_{2}^{D(3)}$ as function of $Q^{2}$ for different values of $\beta$ at fixed $x_{\mathbb{P}}$. The data show a relatively flat $\beta$ dependence and a rising dependence on $Q^{2}$, except at the highest values of $\beta$. This structure is well described by a fit based on DGLAP evolution of the $\beta$ and $Q^{2}$ dependence and a Regge motivated $x_{\mathbb{P}}$ dependence. In this fit, the diffractive parton distributions of the proton are heavily dominated by a large gluon density. The scaling violations of $F_{2}^{D(3)}$ are similar to those of $F_{2}$ when compared at the same $x$ values, except at large $\beta$ where vector meson and other higher twist contributions are expected to play a significant role in the diffractive data.

To extract the Pomeron parton densities we have to assume a factorization of $F_{2}^{D(3)}$ into a Pomeron flux and a Pomeron structure function

$$
F_{2}^{D(3)}\left(x_{\mathbb{P}}, \beta, Q^{2}\right)=f_{\mathbb{P} / p}\left(x_{\mathbb{P}}\right) F_{2}^{\mathbb{P}}\left(\beta, Q^{2}\right) .
$$

The Pomeron flux is taken from formula (3) and integrated over $t$. Under the hypothesis of diffractive factorization, the parton distributions for the Pomeron extracted from $F_{2}^{D(3)}$ are expected to describe diffractive interactions wherever perturbation theory may be applied. The Tevatron data allow such cross check.

At Tevatron the CDF (2001) has measured the diffractive structure function of the antiproton using a method employing two samples of dijet events produced in $p \bar{p}$ collisions at $\sqrt{S}=$ $1800 \mathrm{GeV}$ : a single proton diffractive dissociation sample, and an inclusive sample, collected with a minimum bias trigger. In leading order QCD, the ratio of the diffractive to inclusive cross sections as function of Bjorken $x$ of the struck parton in the antiproton, obtained from the jet kinematics, is equal to the ratio of the corresponding structure functions. This method gives a model independent colour-weighted structure function

$$
F_{j j}^{D}(x)=\left\{g^{D}(x)+\frac{4}{9} \sum_{i}\left[q_{i}^{D}(x)+\bar{q}_{i}^{D}(x)\right]\right\},
$$

where $g^{D}(x)$ and $q_{i}^{D}(x)$ are the antiproton gluon and quark diffractive parton densities. By changing $x$ to $\beta=x / x_{\mathbb{P}}$ the obtained parton distribution can be compared with that calculated using parton densities in Pomeron measured at HERA and the standard Pomeron flux. This comparison is shown in Figure 4. The Tevatron and HERA parton distributions disagree both in shape and normalization by an order of magnitude. Similar sizable disagreement with predictions based on the Pomeron structure measured at HERA and the standard Pomeron flux are reported by $\mathrm{CDF}$ in reactions of $W, b$-quark and $J / \Psi$ diffractive production (Goulianos 2001). Thus, under the factorization hypothesis a Pomeron at the Tevatron has 10 times lower parton density than that at HERA! In addition, a steeply rising SDD cross section given by (3) overshoots the Tevatron measurement of $\sigma_{S D D}$ by a similar factor (Goulianos 1995).

A way out of this contradiction is to revise calculations of the Pomeron flux. It was suggested (Kaidalov 2001) that at Tevatron energies additional parton rescatterings kill rapidity gaps and therefore, effectively suppress the standard Pomeron flux. In this approach a suppression factor is different for diffractive jet production and for inclusive diffraction. Another approach is to renormalize a rapidity gap probability (Goulianos 2000) in order to describe experimentally observed scaling law for diffractive dissociation. A spectacular graphical representation of this scaling law is shown in Figure 5, where the measured spectra $d \sigma^{2} / d t d M_{X}^{2}$ at fixed 
$t$ are plotted as function of $M_{X}^{2}$ for $p p$ collision energies from $\sqrt{s}=14$ to $\sqrt{s}=1800 \mathrm{GeV}$. This data show an independent of $s$ universal behaviour of the differential diffraction cross section. This remarkable universality is highly non-trivial and disagree with $s$ dependence expected from the Regge formula (3). Whatever theoretical approach is used to make corrections to the Regge flux it looks like a construction of a Ptolemaic system. The factorization breaks down such a way that gives rise to a universal scaling law!

\section{Exclusive diffractive meson production}

The HERA collider provides a unique opportunity to observe a rich family of exclusively produced vector mesons in a diffractive reaction.

$$
e+p \rightarrow e^{\prime}+p^{\prime}+V
$$

In $e p$ scattering the photon irradiated by the electron interacts with the Pomeron emitted from proton and may materialize into a meson with quantum numbers of the photon $\left(J^{C P}=1^{--}\right)$.

In Figure 6 the cross section for elastic electroproduction of vector mesons is presented as function of the variable $\left(Q^{2}+M_{V}^{2}\right)$. The data in Figure 6 compile the HERA measurements (H1 2000) at $\sqrt{s_{\gamma p}}=75 \mathrm{GeV}$ scaled by SU(5) factors, according to the quark charge content of the vector mesons, which amount to 1 for the $\rho, 9$ for the $\omega, 9 / 2$ for the $\phi$ and $\Upsilon$ and $9 / 8$ for the $J / \Psi$ meson. Within the experimental errors, the exclusive vector meson production cross sections, including the SU(5) normalization factors, turned out to lie on a universal curve which is close to a simple $\sim 1 /\left(Q^{2}+M_{V}^{2}\right)^{2.2}$ behaviour. Note, that that the ratio of SU(5) factors is in fact can be approximated by ratios of the electronic width $\Gamma_{e e}$ of the vector mesons. Thus, for the photoproduction $\left(Q^{2} \approx 0\right)$ the dependence of the exclusive vector meson cross section is

$$
\sigma_{\gamma p \rightarrow V p} \sim \Gamma_{e e}\left(\frac{1}{M_{V}^{4}}\right)^{1.1} .
$$

The extra power term in (6) and ( 4 ) is taken to be $\alpha_{\mathbb{P}}=1.1$, which agrees with the data within the experimental errors. Interesting to observe that this universality holds true for light $\rho^{0}$, where perturbative calculations are not directly applicable and for heavy quarkonia states, where it is believed that perturbative predictions are straight forward. In addition, at $Q^{2}=0$ the photon interacting with the target proton has a hadronic structure. As found by Block (2001), the rescattering effects are expected to suppress the diffractive $\gamma p$ cross section in a same way as in hadron-hadron collisions. In addition these corrections work differently for low mass vector mesons and for heavy $J / \Psi$ and $\Upsilon$. Remarkable, that after cooking these corrections in a soup from particles of different masses spiced with various flavours of constituent quarks the beautiful and simple scaling law (6) emerges.

It is interesting to point out a close similarity of exclusive diffractive vector meson production in $\gamma p$ to inclusive meson production in hadronic collisions. It was found (Gaisser 1978) that the inclusive cross section for hadroproduction of narrow vector mesons $(\phi, J / \Psi, \Upsilon)$ follow the scaling law

$$
\sigma_{p p \rightarrow V X} \sim \frac{\Gamma_{g g g}}{M_{V}^{4}},
$$


where $\Gamma_{g g g}$ is a hadronic width for the Okubo-Zweig-Iizuka rule violating decays of $J / \Psi$ and $\Upsilon$, and calculated three gluon width for the $\phi$. This scaling law spans over magnitudes of difference in mass, width and production cross section of the mesons. The scaling law (7) was found at that time in low energy $p p$ collisions. I am not aware of any check that this simple relation works at Tevatron energy.

As an exercise, we divide the inclusive production cross sections by the full width of flavourless particles produced at Tevatron energy $\sqrt{s}=1800 \mathrm{GeV}$ and plot this ratio as function of particles mass. Figure 7 shows the compilation of the data, where in order to simplify a direct comparison, the differential production cross section $d \sigma / d y(y=0)$ is used. The data for $\Upsilon, W$ and $Z$ come from CDF (1995), D0 (1999), and CDF (2000a) correspondingly. The data for $\rho, \phi$ and $f_{2}$ are not available directly at Tevatron and were extrapolated from the ISR measurements (Albrow 1979, Drijard 1981). The $J / \Psi$ production cross section was estimated by extrapolation of measured $P_{t}$ spectrum (Sansoni 1996). Wherever the extrapolation was applied, additional $80 \%$ systematic uncertainty is attributed to the data points on the plot in Figure 7. Remarkably, the mesons roughly follow a scaling law similar to (5) and (6)

$$
\frac{d \sigma_{p p \rightarrow M X}}{d y}(y=0) \sim \frac{\Gamma}{M^{4}} .
$$

The narrow $J / \Psi$ and $\Upsilon$ resonances lie above the rest of the particles, though, they follow the universal dependence (8). Could it be pure accidental, that the light hadrons $\rho, \phi$ and $f_{2}$ lie on the same line as heavy and pointlike gauge bosons $W$ and $Z^{0}$ ? To extend the exercise we add to the plot in Figure 7 an area of the expected values $\sigma / \Gamma$ for low mass Higgs boson production at the Tevatron (Carena 2000). It turned out, that expected Higgs production cross section is close to a naive estimate based on the scaling law (8).

Like the mesons produced in diffractive reaction (5) carry quantum numbers of photon, the exclusively produced in DPE mesons have quantum numbers of the Pomeron $\left(I^{G} J^{C P}=\right.$ $\left.0^{+} J^{++}\right)$. Generally, the DPE is one of the most spectacular phenomenon and might be seen as a natural laboratory to study Pomeron-Pomeron interactions. For inclusive DPE reaction it was found (Breakstone 1989) that resulting hadronic system resembles that produced in a single diffractive dissociation. It shows no anomaly in relative yield of pions kaons and protons with respect to that in non-diffractive inclusive hadroproduction, and the differential cross section of DPE process observes $1 / M_{X}^{2}$ behaviour typical for other classes of inelastic diffractive reactions. Further on we will consider the exclusive DPE reactions only.

Experimentally the DPE exclusive state production has been extensively studied in $p p$ collisions at energies up to ISR energies. The available data are limited to light $f_{0}, f_{1}$ and $f_{2}$ mesons. Those mesons are produced at low $|t|$ and its production cross section rises slowly with the collision energy (Kirk 2000). A prominent example of DPE meson production is

$$
p+p \rightarrow p+f_{2}(1270)+p,
$$

with measured cross section at $\sqrt{s}=62 \mathrm{GeV}$ to be $14 \pm 5 \mu \mathrm{b}$ (Breakstone 1986). The DPE cross section rises slowly with $s$ and one could expect a twice larger cross section for exclusive $f_{2}(1270)$ production at Tevatron energy. The Tevatron experiments have large phase space to produce heavy particles in the DPE reaction. Taking a hypothesis that the exclusive DPE meson production observes the scaling law found for inclusive hadroproduction (8) one would expect 
for $\chi_{c}$ DPE production about $3 n b$. This cross section is large enough to be measured at the Tevatron provided a trigger is efficient for low $P_{t}$ muons and electrons from a cascade decay.

The Higgs boson has correct quantum numbers and can be produced exclusively in the DPE reaction

$$
p+p \rightarrow p+H+p
$$

Presently, the theoretical predictions for the $p p \rightarrow p H p$ cross section at the Tevatron vary form about 0.1 to $100 \mathrm{fb}$ for the low mass Higgs. Such big uncertainty in the calculations reflects the difficulty to describe non-perturbative effects associated with the formation of rapidity gaps. We boldly go ahead and apply the scaling law for the exclusive Higgs production at Tevatron

$$
\sigma(p p \rightarrow p H p)=\sigma\left(p p \rightarrow p f_{2} p\right) \frac{\Gamma_{H} M_{f}^{4}}{\Gamma_{f} M_{H}^{4}} \approx 10 f b .
$$

If this estimate will come to be correct, the $p p \rightarrow p H p$ reaction give a unique chance to discover low mass Higgs at Tevatron. Recently, it has been shown (Albrow 2000) that a measurement of outgoing $p$ and $\bar{p}$ momenta in the reaction (9) will allow to reach unprecedented Higgs mass resolution of $250 \mathrm{MeV}$ by calculating a missing mass. This challenging measurement will require a precise monitoring of the beam parameters, probably, using high statistics elastic $p \bar{p}$ scattering. Given expected for Tevatron Run II luminosity of $15 \mathrm{fb}^{-1}$ and average acceptance of $50 \%$ the total number of reconstructed $\mathrm{pH} p$ events is 75 at a negligible background. A relatively low cost of detector modification required for this measurement makes this measurement very attractive in the future. The Pomeron physics was before and is now a rich source of information about QCD vacuum and non-perturbative phenomena. I believe, it will remain an experimental field of spectacular observations.

\section{Acknowledgements}

The work was partially supported by Russian Foundation for Basic Research, grant RFBR-01-02-16431. 


\section{References}

Akerlof, C.W., et al, "Hadron-proton elastic scattering at 50, 100 and $200 \mathrm{GeV} / \mathrm{c}$ momentum”, Phys.Rev. D14, 2864 (1976).

Albrow, M.G. and Rostovtsev, A., "Searching for the Higgs boson at hadron colliders using the missing mass method", FERMILAB-PUB-00-173, e-Print Archive: hep-ph/0009336, (2000).

Albrow, M.G., et al, "Inclusive $\rho^{0}$ production in $p p$ collisions at the CERN ISR", Nucl.Phys., B155, 39 (1979).

Bjorken, James D., "Hard diffraction and deep inelastic scattering", Talk given at International Workshop on Deep Inelastic Scattering and Related Subjects, Eilat, Israel, 6-11 Feb 1994. Published in Eilat DIS Workshop, 151 (1994).

Block, M.M. and Halzen, F., "Survival Probability of Large Rapidity Gaps in $\bar{p} p, p p, \gamma p$ and $\gamma \gamma$ Collisions”, Phys.Rev. D63, 114004 (2001).

Bolotov, V.N., et al, "Negative pion charge exchange scattering on protons in the momentum range $20-50 \mathrm{GeV} / \mathrm{c}$ ", Nucl.Phys. B73, 365 (1974).

Breakstone, A., et al, "Inclusive Pomeron-Pomeron interactions at the CERN ISR", Z.Phys., C42, 387 (1989).

Breakstone, A., et al, "Production of the $f^{0}$ meson in the double Pomeron exchange reaction $p p \rightarrow p p \pi^{+} \pi^{-}$at $\sqrt{s}=62 \mathrm{GeV}^{\prime}$, Z.Phys., C31, 185 (1986).

Carena, M., et al, "Report of the Tevatron Higgs working group", FERMILAB-CONF-00279-T, e-Print Archive: hep-ph/0010338, (2000).

CDF Collab., "Measurement of $d \sigma / d y$ for high mass Drell-Yan $e^{+} e^{-}$pairs from $p \bar{p}$ collisions at $\sqrt{s}=1800$ GeV", Phys.Rev., D63, 011101 (2001).

CDF Collab., "Diffractive dijets with a leading $\bar{p}$ in $\bar{p} p$ collisions at $\sqrt{s}=1800 \mathrm{GeV}$ ", Phys.Rev.Lett. 84, 5043,(2000).

CDF Collab., " $\Upsilon$ production in $p \bar{p}$ collisions at $\sqrt{s}=1800 \mathrm{GeV}$ ”, Phys.Rev.Lett., 75, 4358 (1995).

D0 Collab., "Measurement of $W$ and $Z$ boson production cross-sections", Phys.Rev., D60, 052003 (1999).

Drijard, D., et al, "Production of vector and tensor mesons in proton-proton collisions at $\sqrt{s}=52.5 \mathrm{GeV}^{\prime}$, Z.Phys., C9, 293 (1981).

Donnachie, A. and Landshoff, P.V., “Total cross sections”, Phys.Lett. B296, 227 (1992).

Frautschi, S.C., Gell-Mann, M. and Zachariasen, F, "Experimental consequences of the hypothesis of Regge poles", Phys.Rev. 126, 2204 (1962).

Gaisser, T.K., Halzen, F., and Paschos and E.A., "Hadronic production of narrow vector mesons", Phys.Rev., D15, 2572 (1977). 
Goulianos, K., "The Diffractive structure function at the Tevatron: CDF results", Nucl.Phys.Proc.Suppl. 99A, 37 (2001).

Goulianos, K., “Diffraction: a new approach”, J.Phys., G26, 716 (2000).

Goulianos, K., and Montanha, J., "Factorization and scaling in hadronic diffraction", Phys.Rev., D59, 114017 (1999).

Goulianos, K., "The structure of the pomeron", e-Print Archive: hep-ph/9505310, (1995).

Goulianos, K., "Diffractive interactions of hadrons at high energies", Phys.Rep. 101, 169 (1983).

Gribov, V.N., "Possible asymptotic behavior of elastic scattering", Soviet Phys.-JETP 14, 478 (1961).

H1 Collab., "Measurement of elastic electroproduction of $\phi$ mesons at HERA", Phys.Lett., B483, 360 (2000).

H1 Collab., "Inclusive measurement of diffractive deep-inelastic ep scattering", Z.Phys. C76, 613 (1997).

H1 Collab., "Observation of hard processes in rapidity gap events in gamma-p interactions at HERA”, Nucl.Phys., B435, 3 (1995).

Ingelman, G. and Schlein, P., "Jet structure in high mass diffractive scattering", Phys.Lett. 152B, 256 (1985).

Kaidalov, A.B., Khoze, V.A. Martin, A.D. and Ryskin, M.G., "Probabilities of rapidity gaps in high energy interactions", e-Print Archive: hep-ph/0105145, (2001).

Kirk, A., "Resonance production in central $p p$ collisions at the CERN Omega Spectrometer”, Phys.Lett., B489, 29 (2000).

Newman, P. "Measurement of the diffractive structure function at HERA", Talk given at the International Europhysics Conference on High Energy Physics, Budapest, July (2001).

Sansoni, A., "Quarkonia production at CDF", Talk given at 12th International Conference on Ultra-Relativistic Nucleus-Nucleus Collisions (Quark Matter 96), Heidelberg, Germany, 2024 May 1996., Published in Nucl.Phys., A610 373, (1996). 


\section{Figures}

Figure 1: Regge trajectories for the charge exchange (a) and elastic scattering (b) reactions.

Figure 2: A spectrum of the rapidity gap width distribution measured at H1 (1995).

Figure 3: Dependence of $F_{2}^{D(3)}$ on $Q^{2}$ for different values of $\beta$ at fixed $x_{\mathbb{P}}$. The data are taken from (Newman 2001).

Figure 4: A comparison of the colour-weighted Pomeron parton densities as measured at HERA (upper curves) and at Tevatron (the data points below).

Figure 5: Double differential SDD cross section for $p(\bar{p})+p \rightarrow p(\bar{p})+X$ at $t=-0.05 \mathrm{GeV}^{2}$ and $\sqrt{s}=14,20,546$ and $1800 \mathrm{GeV}$.

Figure 6: A Compilation of HERA measurements of the total cross sections $\sigma\left(\gamma^{*} p \rightarrow V p\right)$ as function of $\left(Q^{2}+M_{V}^{2}\right)$ for elastic $\rho, \omega, \phi, J / \Psi$ and $\Upsilon$ meson production at the fixed $\sqrt{s_{\gamma^{*} p}} \equiv$ $W=75 \mathrm{GeV}$. The measured cross sections were scaled by $\mathrm{SU}(5)$ factors according to the quark charge content of the vector meson. The insert presents a ratio of the scaled $\omega, \phi$ and $J / \Psi$ cross sections to the parameterization curve.

Figure 7: The $d \sigma / d y(y=0)$ cross section scaled by the values of the full width as function of the particle mass for $\rho, \phi, f_{2}(1270), J / \Psi, \Upsilon, W^{+}$and $Z$ particles produced in $p p$ collisions at $\sqrt{s}=1800 \mathrm{GeV}$. The curves illustrate $1 / M^{4}$ dependence. The area of expected values $\sigma / \Gamma$ for low mass Higgs boson production at Tevatron is shown with a large filled circle. 

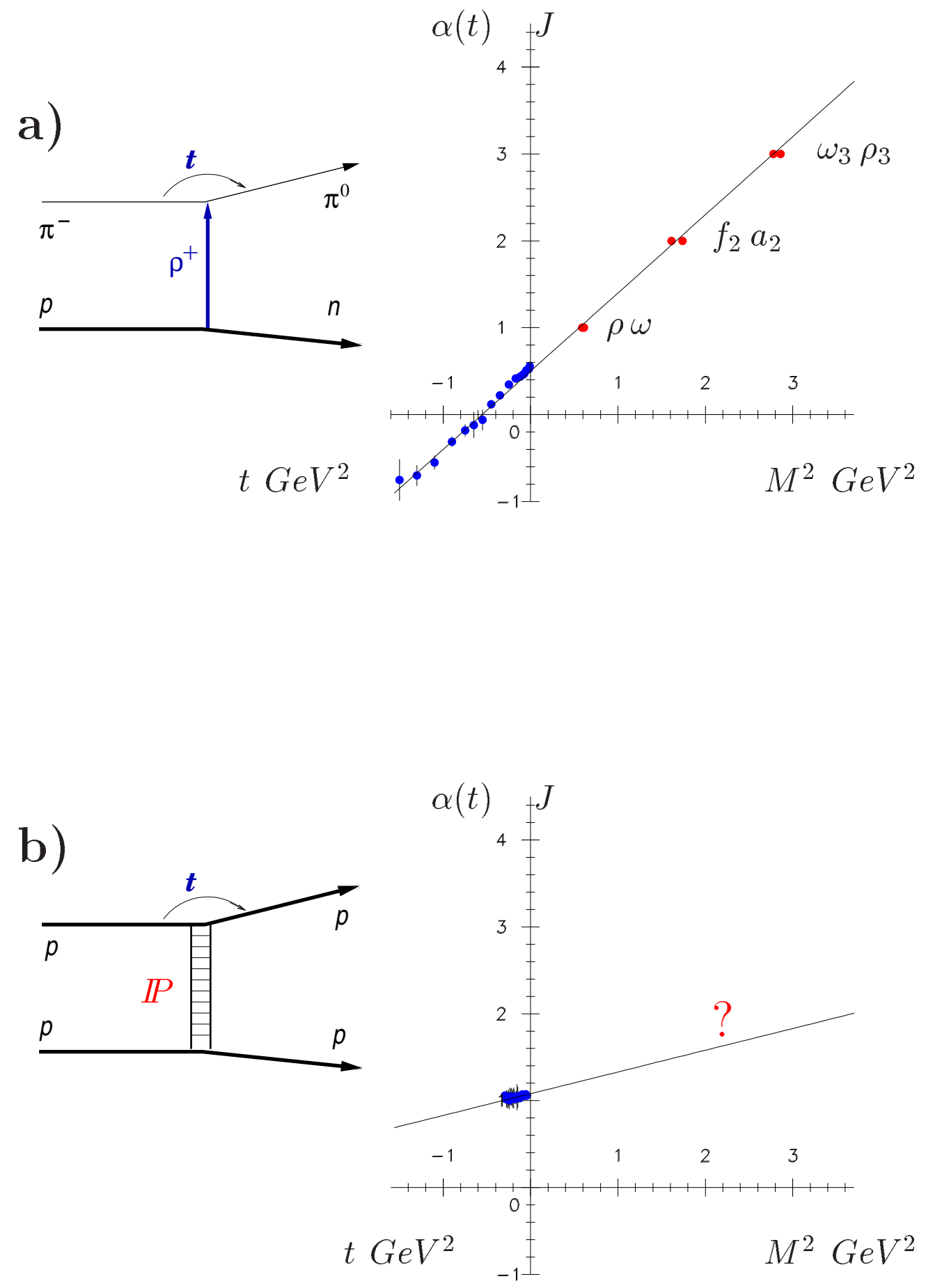

Figure 1 


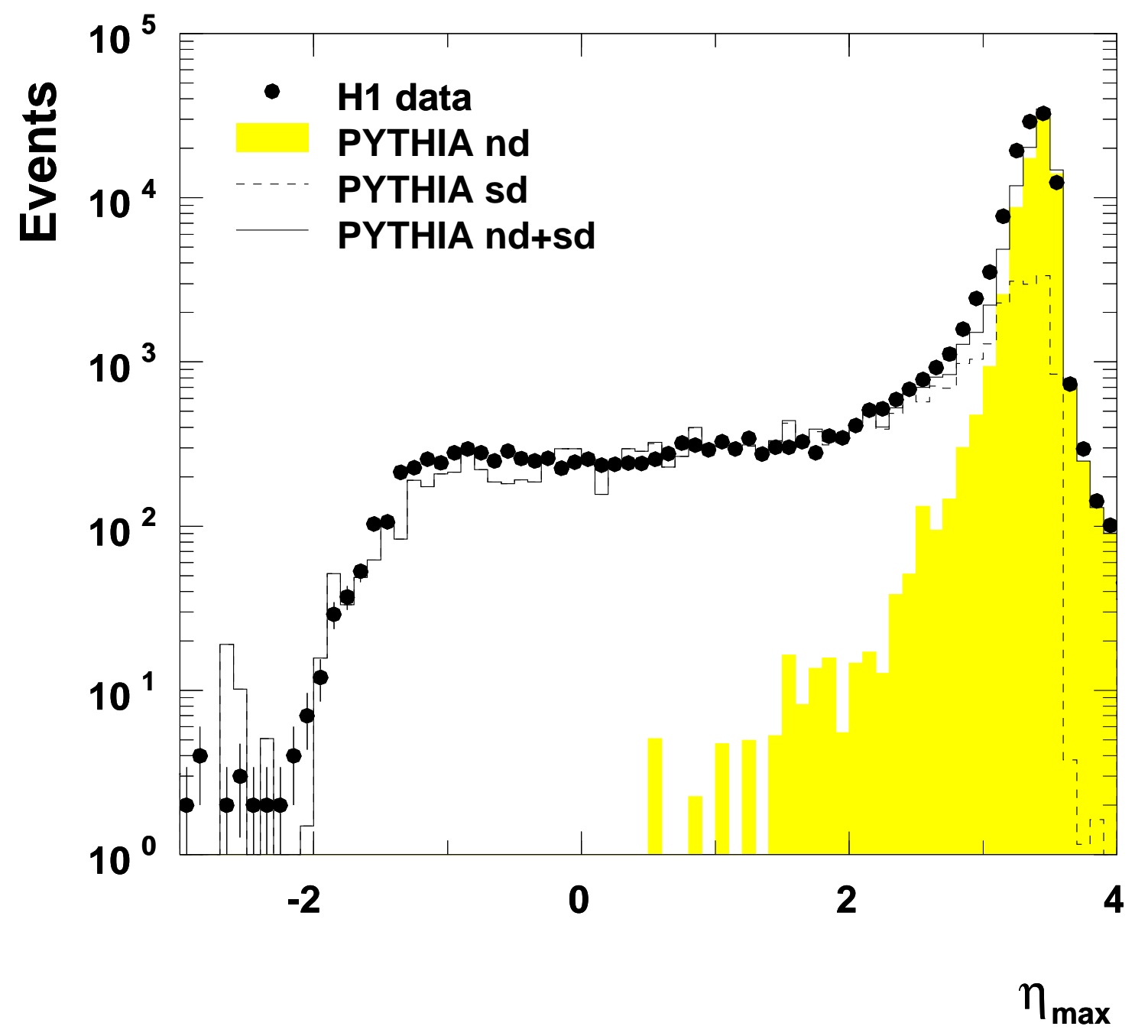

Figure 2 


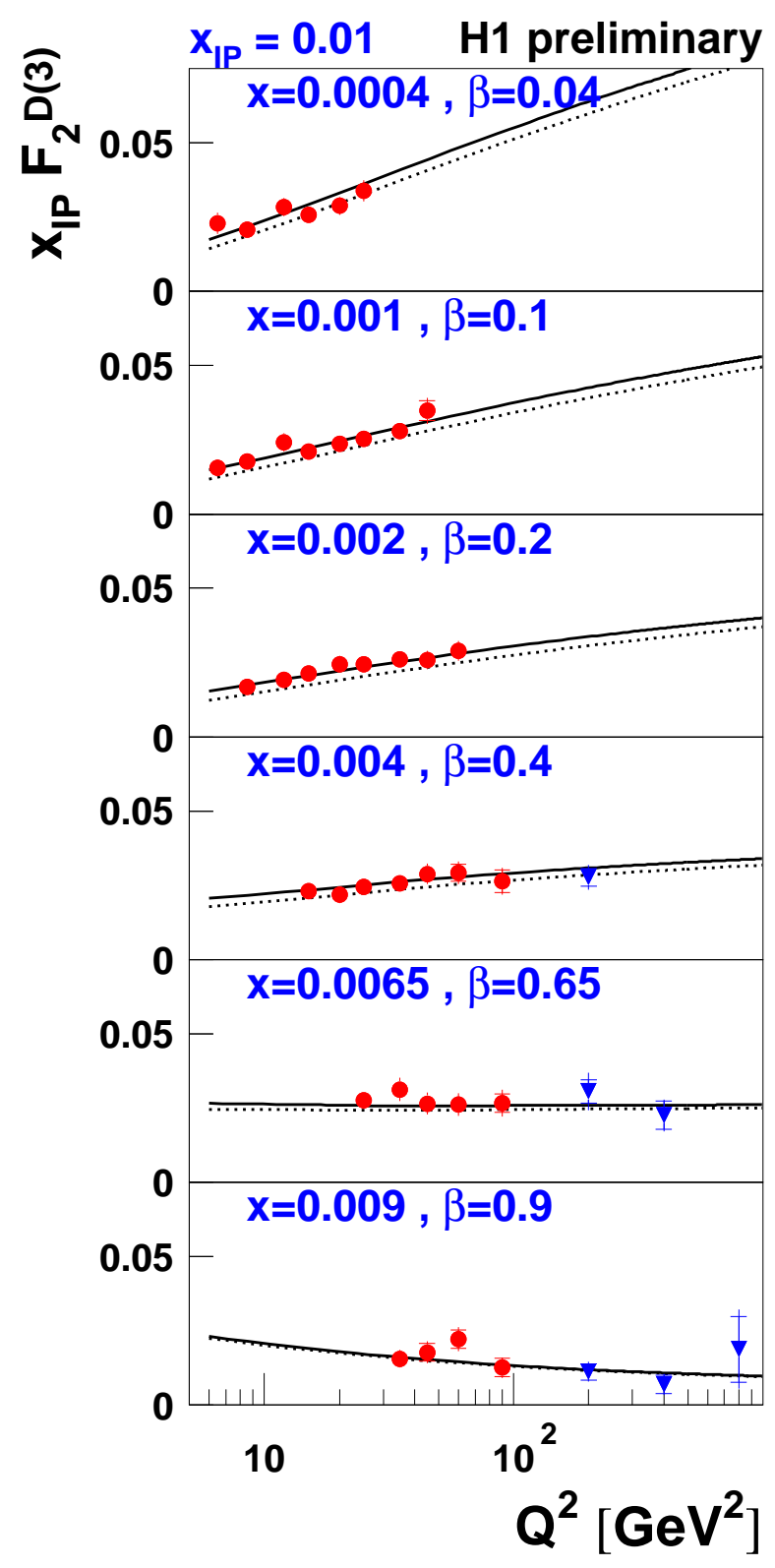

Figure 3 


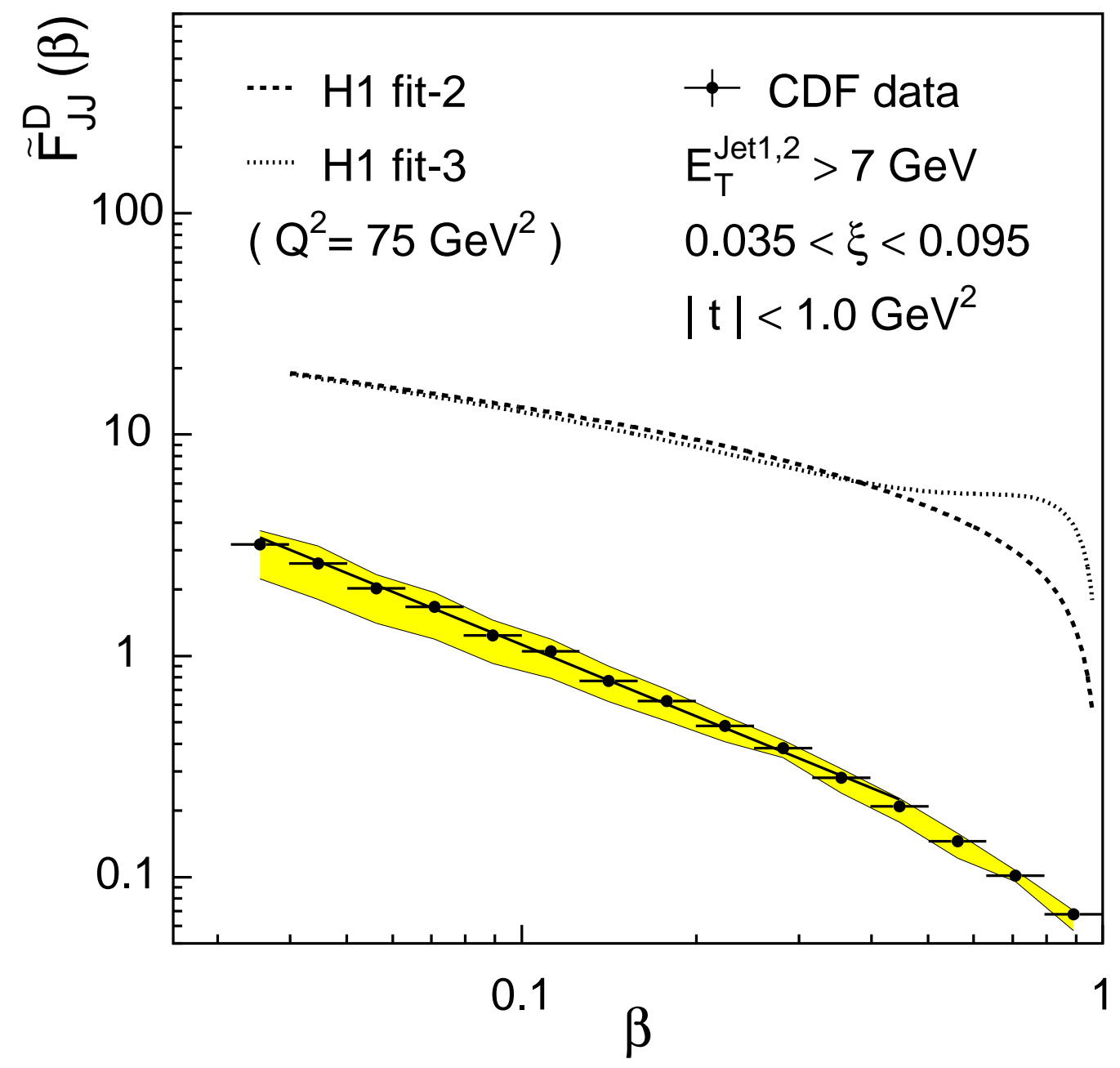

Figure 4 


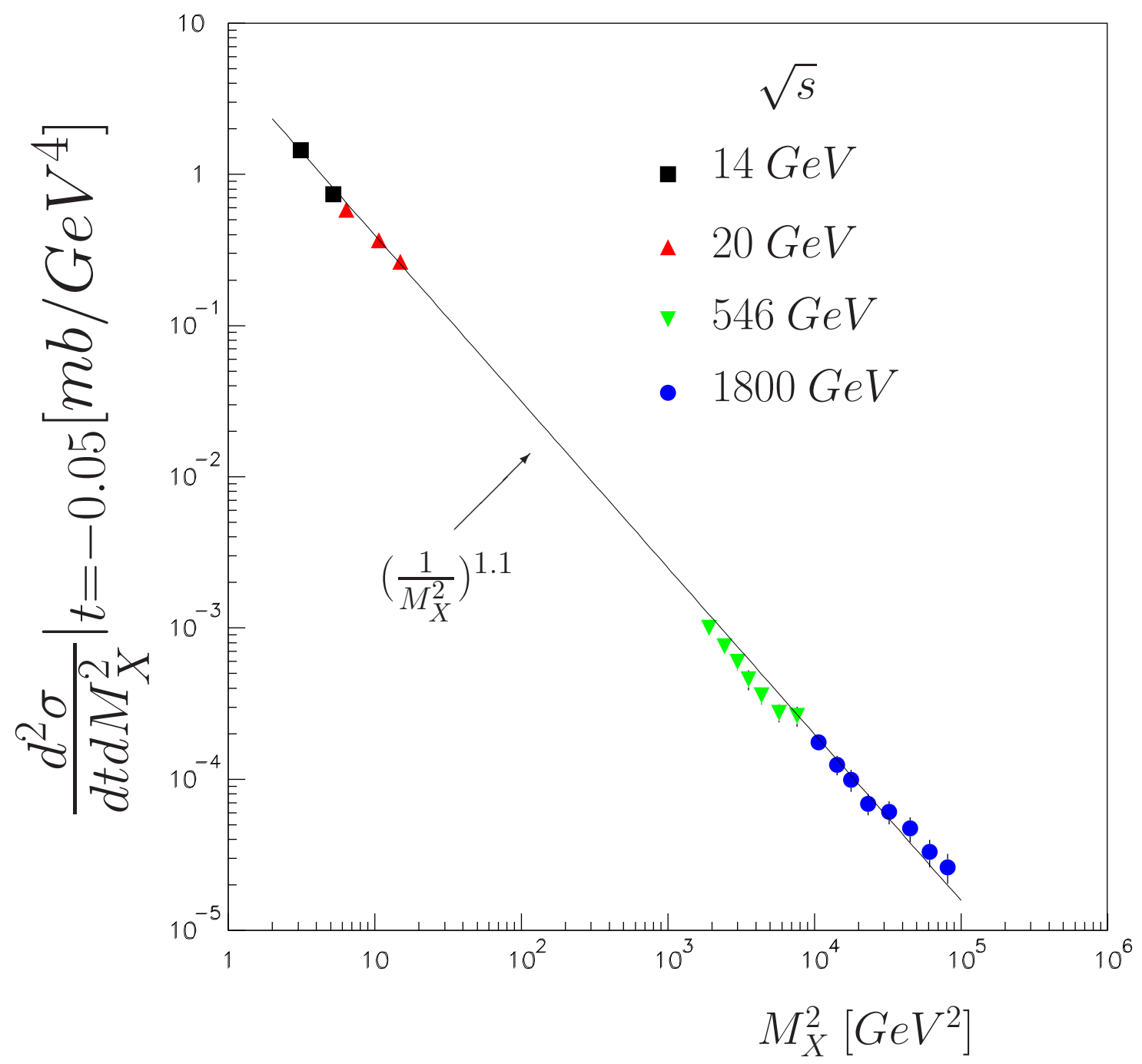

Figure 5 


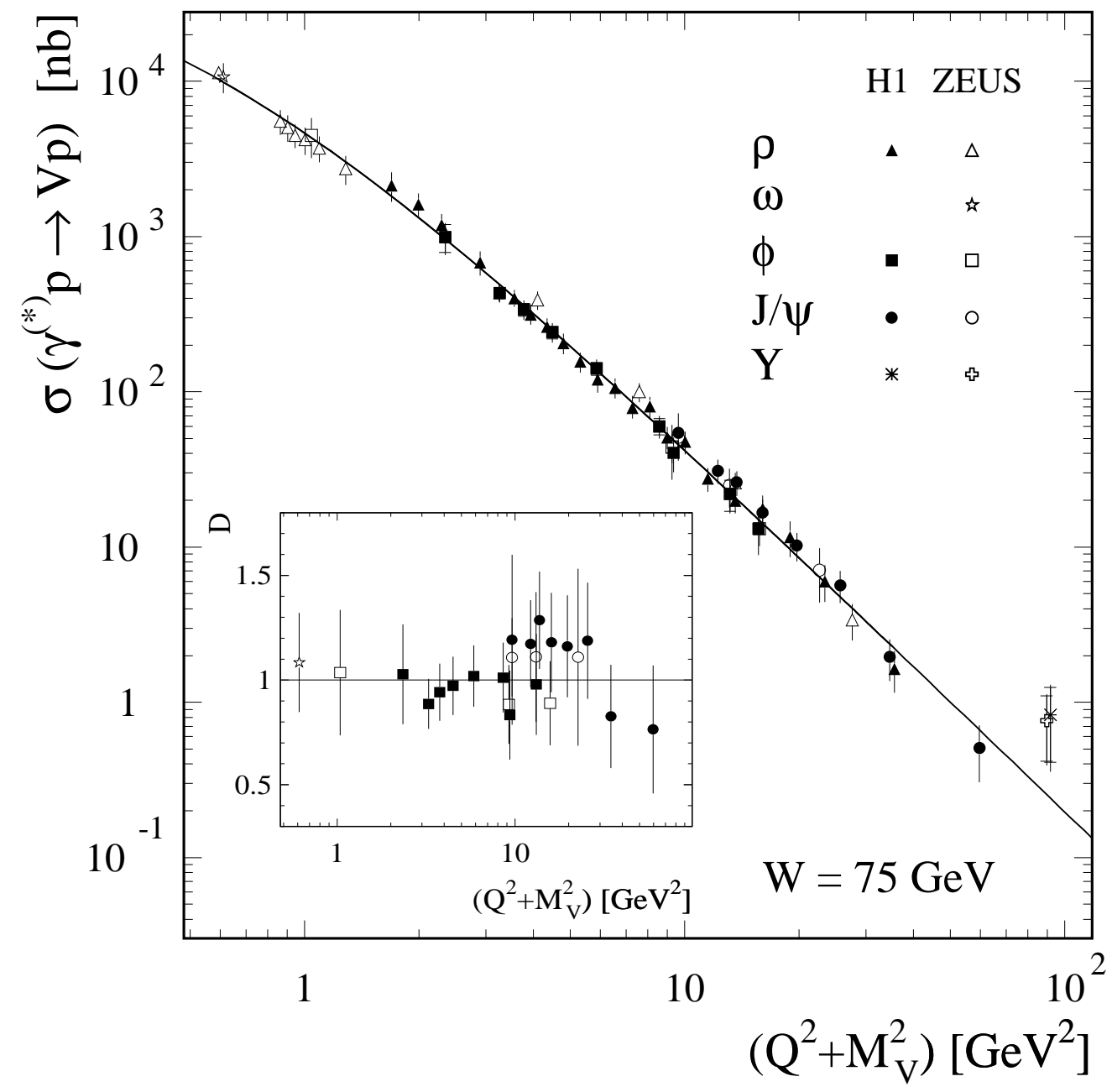

Figure 6 


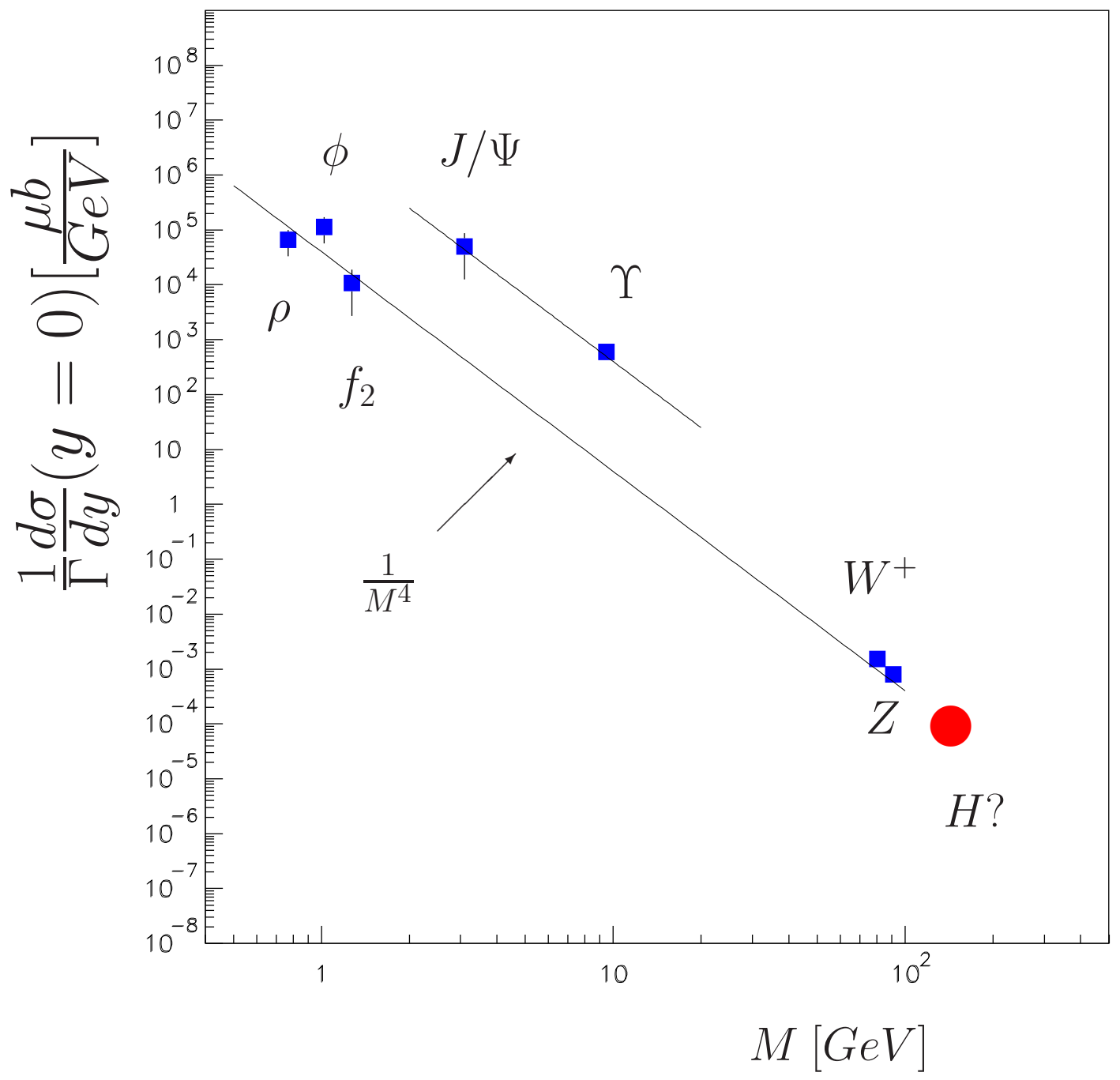

Figure 7 